\title{
Pica - Mind over matter: A case report
}

\author{
SS Ratnatunga, R Hanwella, VA de Silva
}

\section{Abstract}

The geneses, treatment and outcome of pica remains the subject of much debate. Data regarding pica in adults and in schizophrenia is limited. We present the case of a forty-year old male who presented with subacute intestinal obstruction. Having met the diagnostic criteria for schizophrenia, he subsequently developed symptoms which enabled a diagnosis of pica. Initially the pica symptoms were as a response to the command hallucinations, but later persisted in the absence of hallucinations. Pica can be potentially life-threatening, depending on the substance ingested and the complications that follow, as was the case in this patient. Risperidone, olanzapine or electro convulsive therapy did not have any impact on his symptoms of pica. Clozapine was commenced and a dramatic response was observed, with the pica symptoms completely resolving within 10 days - thus revealing a new facet to the already versatile therapeutic effects of clozapine.

Key words: pica, schizophrenia, clozapine

SL J Psychiatry 2016; 7(2): 26-28

\section{Case Report}

M was a forty-year old unskilled labourer from Colombo. He was referred from a surgical ward following conservative management of sub acute intestinal obstruction. He had presented with abdominal pain and constipation for three days. Imaging revealed that there were radioopaque material in his intestines (Figure 1). Three months prior to his presentation $\mathrm{M}$ had developed poor sleep and suspiciousness of family members. He believed that his wife and twelve-year old son were plotting to kill him. He had limited contact with family and refused meals prepared by his wife. There had been frequent absenteeism from work. These symptoms were followed by the development of voices of unseen males discussing him in a derogatory manner in the third person. The voices commanded him to swallow metal objects, which he resisted at first, then complied with, as he feared the consequences of not obeying. He initially swallowed a small nail and then other small metal objects such as pins, clips, coins and blades. Subsequently he swallowed metal objects in the absence of such commands. He spent the next few weeks confining himself to his room, his self care and sleep further deteriorated and he started swallowing metal objects on a daily basis. He developed constipation and abdominal pain, which worsened over the course of three days. He admitted himself to the surgical casualty with severe abdominal pain.

This was M's first presentation to psychiatric services. He did not experience low mood, anhedonia or reduced self-esteem. The ingestion of metal objects were not in response to ideas of self harm. He did not believe his body or internal organs were non functional. There were no recurrent repetitive intrusive thoughts, nor any compulsions. There were no episodes of binge eating, fear of gaining weight or excessive self-monitoring.
There was no family history of psychiatric illness and no childhood history of eating non-nutritive substances. There was no substance use or a forensic record. There was no past head injury or history of cerebral infections. He was employed as a labourer in the local municipal council. He lived with his wife and twelve-year old son in a suburban area of Colombo.

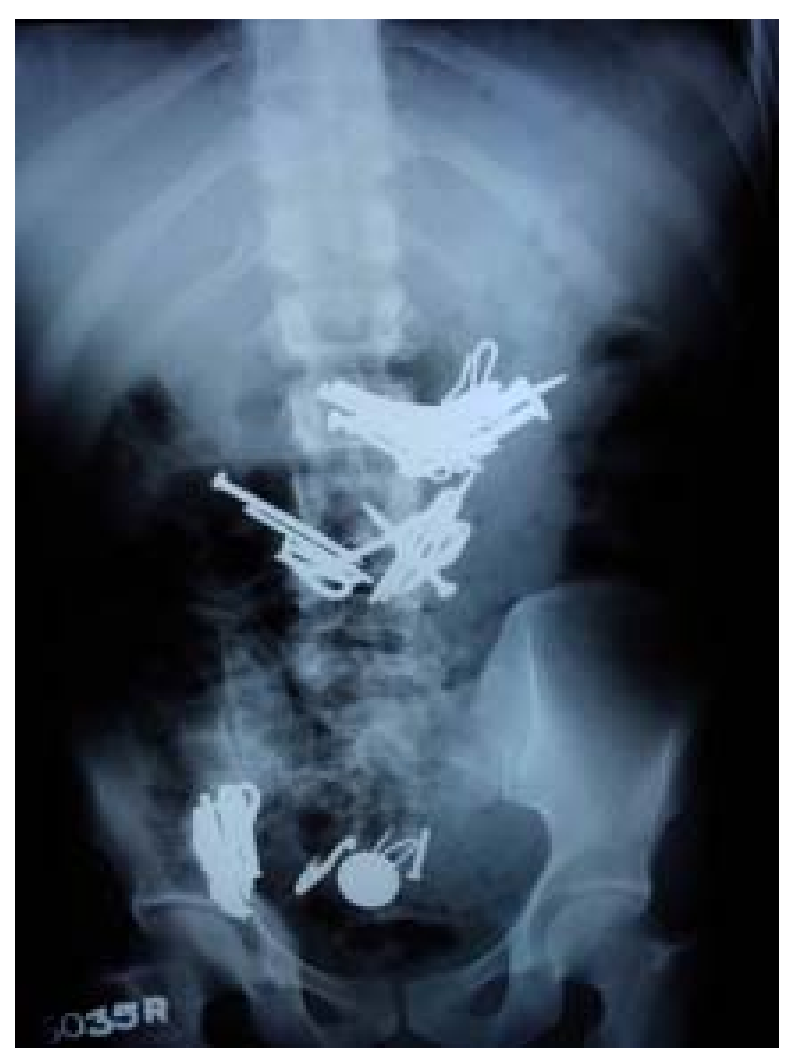

Figure 1. X-ray abdomen showing ingested radio opaque material. 


\section{Mental state examination}

He was a thin built, withdrawn male, with poor self-care and poor eye contact. Speech was hesitant and monotonous. There was no evidence of formal thought disorder. Mood was anxious with poor reactivity. There were no ideas of suicide or homicide. There were delusions of persecution; a firm belief that his wife and son are plotting to kill him. He also had delusions of reference - a firm belief that there was special reference to him in television and radio programs. There were no delusions of infidelity, control, nihilism or thought alienation. There were second and third person auditory hallucinations; voices talking to him and discussing him in the third person. At the time of examination there were no command hallucinations or other perceptual disturbances. Orientation, attention and concentration, short and long-term memory was intact. He had poor insight into his illness. Extended cognitive functions were normal.

IQ assessment: TONI-3 (test of non verbal IQ) was normal.

\section{Physical examination}

BMI- $20.2 \mathrm{~kg} / \mathrm{m}^{2}$, respiratory, cardiovascular and nervous system examination were normal. Examination of the abdominal system revealed a flat abdomen, with mild tenderness on palpation, no masses, bowel sounds were present and normal in intensity. Per rectal examination revealed an empty rectum.

\section{Investigations}

Hematology: WBC $-10 \times 10^{3}$, Hemoglobin -11.8 g/dL, Platelets $-293 \times 10^{3}$, Serum sodium $-138 \mathrm{mmol} / \mathrm{L}$, Serum potassium - $4.8 \mathrm{mmol} / \mathrm{L}, \mathrm{AST}-15 \mathrm{U} / \mathrm{L}, \mathrm{ALT}-30 \mathrm{U} / \mathrm{L}$, Fasting blood sugar $-5 \mathrm{mmol} / \mathrm{L}$, Total cholesterol - 5.1 $\mathrm{mmol} / \mathrm{L}$, Serum albumin - $42 \mathrm{~g} / \mathrm{L}$, Chest x-ray - normal, ECG - sinus rhythm, 2D Echocardiogram - ejection fraction $-60 \%$, no wall motion abnormalities, no valve abnormalities, CT Brain - normal, X-ray-Abdomen multiple radio opaque objects in the intestines (Figure 1).

\section{Management}

The surgical team observed him closely with serial abdominal x-rays. The patient was given adequate hydration and laxatives. Psycho-education of the patient and family was carried out and the risks of swallowing metal objects were explained.

The baseline PANSS (positive and negative syndrome scale) score was - P24, N29, G59. He was treated with risperidone $8 \mathrm{mg} /$ day for two weeks and the PANSS score became-P21, N27, G53. The pica symptoms continued with the same intensity.
Given the poor response to treatment, medication was changed from risperidone to olanzapine $20 \mathrm{mg} /$ day. Iron and zinc supplements were started. The psychotic and negative symptoms improved whilst the pica continued. The PANSS score was P14, N16, G43.

Because there was inadequate response, it was decided to augment olanzapine $20 \mathrm{mg}$ with ECT. After a course of four ECTs and $20 \mathrm{mg}$ of olanzapine, the pica symptoms persisted.

After initial investigations clozapine was started. Clozapine was titrated up to $150 \mathrm{mg}$. The symptoms of pica resolved with further improvement in the PANSS scores. Three months in to follow up, he is free of pica symptoms and devoid of psychotic symptoms. The patient is currently maintained on clozapine $150 \mathrm{mg} /$ day.

\section{Discussion}

The DSM-5 defines pica as the persistent eating of nonnutritive, non-food substances over a period of at least 1 month; inappropriate to the developmental level of the individual (1). In the spectrum of eating disorders pica may be viewed as a derailment of eating behaviour. The clinical consequences vary from intoxication, nutritional deficiencies, parasitosis, formation of bezoars and intestinal obstruction (2). The prevalence of pica is unclear. Among individuals with intellectual disability, the prevalence appears to increase with the severity of the condition. The onset of pica is usually in childhood; however onset in adults is most commonly associated with intellectual disability, pregnancy and other mental disorders.

Eugen Bleuler described eating disorders in schizophrenia about a century ago. However, to date, they remain poorly understood. Binge eating disorders and night eating syndromes are often reported in patients with schizophrenia, the prevalence being approximately $10 \%$. Anorexia nervosa appears to affect among 1 to $4 \%$ of patients (3). The clearest message from this data is that vigilance for eating disorders in patients with schizophrenia is essential. Antipsychotics commonly used in the treatment of schizophrenia have also been implicated in binge eating disorder and compulsive eating (4).

In practice many combinations of eating disorders and schizophrenia are possible. Eating disorders can coexist with psychotic symptoms, they can preclude a psychotic disorder, be a response to them or occur independently (5). These possibilities were considered in this patient as well. Even though initially the pica symptoms were in response to command hallucinations, later on they persisted in the absence of such hallucinations. Thus it appeared likely that as the illness continued, the pica symptoms occurred independently of the psychotic symptoms. 
The pathophysiology of pica remains elusive. A meta analysis in 2015 revealed that pica is significantly associated with low haemoglobin, haematocrit and plasma zinc (2). This patient's indices for haemoglobin and haematocrit were normal. As measuring plasma zinc was not feasible, he was started on micronutrient supplements.

Pica remains a clinical diagnosis. Abdominal x-rays, ultrasound and other scanning methods may reveal obstructions related to pica. Blood tests and other laboratory tests can be used to ascertain levels of poisoning (e.g., heavy metals) or the nature of infection.

Clozapine has been proven to be effective in resistant schizophrenia, preventing aggression in psychotic patients and suicide and in the treatment of resistant mania. There have been a few case reports reporting the beneficial effects of clozapine in the treatment of pica (4). M's response to clozapine was dramatic. At the time he was started on clozapine, the pica symptoms were not occurring in response to hallucinations, and his psychotic symptoms had greatly reduced compared to his initial presentation. Hence it appears likely that the effectiveness of clozapine in the treatment of pica in this patient, was due to direct effects on the symptoms of pica, rather than secondary to improvement of psychotic symptoms. This case report highlights another possible addition to the unique properties of clozapine.

\section{Conflicts of interest}

None declared
SS Ratnatunga, University Psychiatry Unit, National Hospital of Sri Lanka

R Hanwella, Department of Psychological Medicine, Faculty of Medicine, University of Colombo

VA de Silva, Department of Psychological Medicine, Faculty of Medicine, University of Colombo

Corresponding author: SS Ratnatunga

Email: suhashini_sri@yahoo.com

\section{References}

1. American Psychiatric Association. Diagnostic and statistical manual of mental disorders. 5th ed. Arlington, VA: American Psychiatric Publishing, 2013.

2. Miao D, Young SL, Golden CD. A meta-analysis of pica and micronutrient status. Am J Hum Biol 2015; 27(1): 84-93.

3. Kar SK, Kamboj A, Kumar R. Pica and psychosis - Clinical attributes and correlations: A case report. J Family Med Prim Care 2015; 4(1): 149-150.

4. Rohde J, Claussen MC, Kuechenhoff B, Seifritz E, Schuepbach D. Combined symptomatology of psychosis, pica syndrome, and hippocampal sclerosis: A case report. Int J Eat Disord 2013; 46: 89-91.

5. Kouidrat Y, Amad A, Lalau JD, Loas G. Eating disorders in schizophrenia: implications for research and management. Schizophr Res Treatment 2014; 2014: Article ID 791573. 